
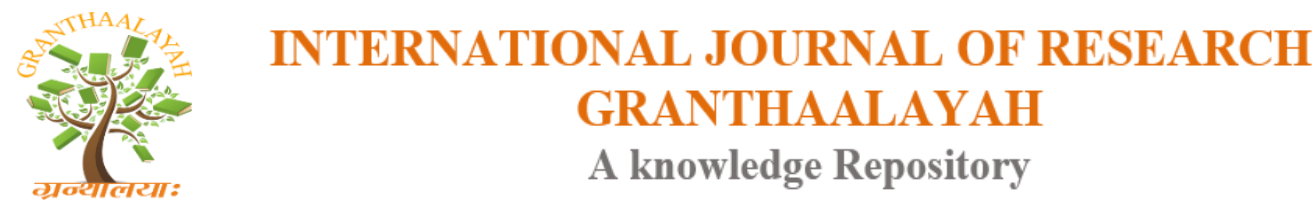

Science

\title{
A STUDY ON THE RELATIONSHIP AMONG ZOOPLANKTONS AND DIFFERENT ABIOTIC FACTORS AT BARDA BANDHARAN WETLAND, KODINAR
}

\author{
Mehul P Dave ${ }^{1}$, Nikul B. Chavda ${ }^{2}$ \\ ${ }^{1}$ Bhakta Kavi Narsinh Mehta University, Junagadh, India \\ ${ }^{2}$ Shri Mahila Arts and Science College, Simar, India
}

\begin{abstract}
Wetland ecosystems are among the most productive ecosystems in the biosphere. Wetland ecosystem supports the growth of Biodiversity. Hydrology of wetland water gives idea about physical and chemical properties of water and their relationship with ecosystem Biodiversity. In present study we investigate relation between Abiotic factors on Zooplankton Diversity. Phytoplankton is integral components of freshwater wetlands, which significantly contribute towards succession and dynamics of zooplankton and fish.

In preset study we have investigated Zooplankton Diversity fluctuation with changing Abiotic factors during Pre, Middle and post winter during 2016-17 of Barda Bandharan (Temporary wetland) near Barda Village, Kodinar, Gujarat.
\end{abstract}

Keywords: Physico-Chemical Parameter; Zooplankton; Phytoplankton; Temporary Wetland.

Cite This Article: Mehul P Dave, and Nikul B. Chavda. (2018). "A STUDY ON THE RELATIONSHIP AMONG ZOOPLANKTONS AND DIFFERENT ABIOTIC FACTORS AT BARDA BANDHARAN WETLAND, KODINAR." International Journal of Research Granthaalayah, 6(4), 23-35. https://doi.org/10.29121/granthaalayah.v6.i4.2018.1473.

\section{Introduction}

Wetland ecosystems are among the most productive ecosystems in the biosphere. Wetlands receive surface water inputs from streams (surface run off), precipitation and overland flow and subsurface water inputs from surface infiltration, stream zones and ground water. These different inputs are important to wetland productivity because they contain markedly different quantities of transported nutrients (1) and organic matter (2). Wetlands are recognized as ecosystems that harbor high biological diversity, provide sustenance for millions of people and face ongoing threats as results of human activities throughout the world (3). As ecosystems, wetlands are highly volatile being particularly vulnerable to environmental fluctuations. Although wetland biodiversity constitutes a significant portion (e.g., 15-20\%), of the total biodiversity of the Indian Subcontinent (4) studies of wetland ecosystems are limited (5). increasing anthropogenic interventions influence in and around aquatic systems and their catchment areas have contributed to a larger extent towards 
deterioration of water quality leading to accelerated eutrophication. The hydro geochemical characteristics and phytoplankton biomass of water bodies are not constant and fluctuate with seasonal variation as well degree of pollution (6).

Phytoplanktons are integral components of freshwater wetlands, which significantly contribute towards succession and dynamics of zooplankton and fish (7).Community structure, dominance and seasonality of phytoplankton in tropical wetlands are highly variable and are functions of nutrient status, water level, morphometry of the underlying substrate and other regional factors (8). Phytoplankton's form the main producers of an aquatic ecosystem which control the biological productivity.

The zooplankton is divided into two groups. Temporary plankton consists of planktonic eggs and larvae of members of the benthos and nekton, permanent plankton includes all animals that live their complete life cycles in a floating state and the temporary plankton particularly abundant in coastal areas, it is characteristically seasonal in occurrence, though variations in spawning time of different species ensure its presence in all seasons. They are absent in fresh water. The ciliate protozoans are represented mainly by the tintinnids, which are between 20 and 640 microns in size and sometimes occur in vast numbers. Oysters, mussels, other marine bivalves and snails begin life as planktonic larvae. The wing snails (Pteropoda) spend their entire life cycles as plankton.

\begin{tabular}{|l|l|}
\hline Sr.No & Barda Bandharan Site Location \\
\hline 1 & N 204659.4, E 0703921.1 \\
\hline 2 & N 204659.7, E 0703927.6 \\
\hline 3 & N 204600.3, E 0703927.6 \\
\hline
\end{tabular}

\section{Material and Method}

\subsection{Sample Collection Points}

Three sample points selected at Barda Bandharan (Sampling Points) with specific GPS location and suitable depth and surface. Sample Collected in plastic bottle (nonmetallic, free-flushing sample recommended for general purpose of water sampling)

5 liter samples Collected for physicochemical analysis approximately less than 2 feet of river water. Time and temperature measured and transferred all sample as soon as possible to laboratory for study further testing. Temperature range between 18 to $21{ }^{0} \mathrm{C}$ of samples (Winter Period)

Collected 5 liter water for chlorophyll estimation in clean bucket to reduce heterogeneity than 1 liter sample was taken from bucket in an opaque plastic bottle for chlorophyll and carotenoids estimation. Plankton sample were collected by using plankton net of $20 \mathrm{um}$ mesh size. 


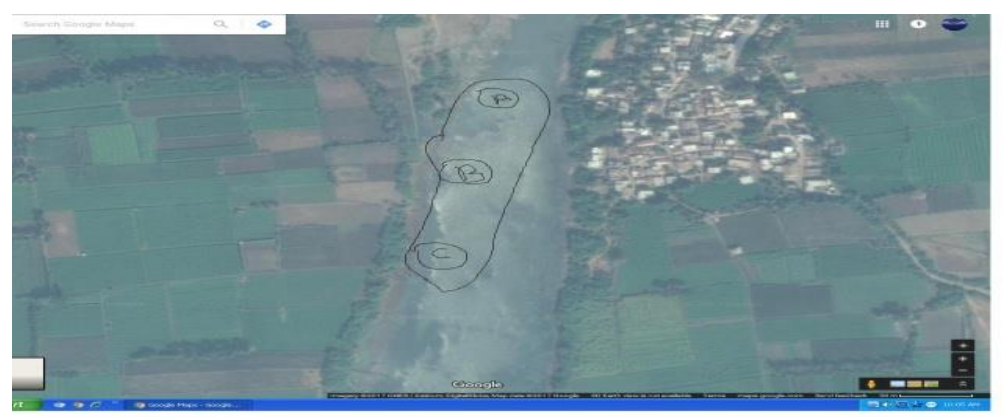

\subsection{Sample Collection for Zooplankton Analysis}

Collected 1 liter river water sample from three collection site with Plankton net ( 0.20 microne). After collection of river water samples it's transferred as soon as possible to laboratory for Analysis. Add 4\% formalin solution and stay it for $48 \mathrm{hrs}$, after incubation time period drop count Method used for identified plankton diversity.

\section{Physicochemical Analysis}

Primary Examination has done Base on Physical examination of water sample by Color, odor and turbidity. $\mathrm{pH}$ and Conductivity measured by $\mathrm{pH}$ meter and Conductivity meter.

\subsection{Estimation of Total solid (T.S.)}

Porcelain dish is used for this method; Heat it for 103 to $105 \mathrm{C}$ for $1 \mathrm{hrs}$. Store and cool dish in desiccators until needed weight immediately before use. (Pre weight)Shake the water sample very well and add $100 \mathrm{ml}$ of it in to evaporating Petri dish. Put evaporating dish in to oven at 103 to $105 \mathrm{C}$ for overnight. Next day take out it from oven and cool it in desiccators dish would be having dried residues in it. Measure the weight of evaporating dish. (Post weight)Put the data or pre weight and post weight of the dish in following equation and calculate the amount of total solid present in the sample.

Calculation: mg total solids/L= (A-B).1000/Sample volume (ml)

Where,

$\mathrm{A}=$ post weight of dish (weight of dried residues +dish $\mathrm{mg}$ )

$\mathrm{B}=$ Pre weight (weight of dish $\mathrm{mg}$.)

\subsection{Estimation of Total Dissolved Solid (T.D.S.)}

Porcelain dish is used for this method; Heat it for 103 to $105 \mathrm{C}$ for $1 \mathrm{hrs}$. Store and cool dish in desiccators until needed weight immediately before use. (Pre weight)Shake the water sample very well and add $100 \mathrm{ml}$ of it in to filtration device that is having glass fiber on it. Apply vacuum and filter out $100 \mathrm{ml}$ of sample. Collect the filtrate in to evaporating dish. Put evaporating Petri dish in to oven at 103 to $105 \mathrm{C}$ for overnight. Next day take out it from oven and cool it in desiccators dish would be having dried residues in it. Measure the weight of evaporating dish. (Post weight) Put the data of pre weight and post weight of the dish in following equation and calculate the amount of total solid present in the sample. 
Calculation: $\mathrm{mg}$ total dissolved solid/L= $(\mathrm{A}-\mathrm{B}) .1000 /$ sample volume $(\mathrm{ml})$

Where,

$\mathrm{A}=$ Post weight of dish (weight of dried resdues +dish, mg)

$\mathrm{B}=$ pre weight (weight of dish, $\mathrm{mg}$ )

\subsection{Estimation of Chloride in Water Sample}

Sample preparation: Take $100 \mathrm{ml}$ of sample in $250 \mathrm{ml}$ conical flask. If chlorine is higher in the sample, dilute the sample and then take $100 \mathrm{ml}$ of diluted sample. If the sample is highly colored $\operatorname{add} 3 \mathrm{ml} \mathrm{Al}(\mathrm{OH})_{3}$ suspension, mix, settle and filter.

Titration: Set the $\mathrm{pH}$ of the sample in the range of 7-10 with the help of $\mathrm{H}_{2} \mathrm{SO}_{4} / \mathrm{NAOH}$.

Add $1 \mathrm{ml} \mathrm{K} 2 \mathrm{CrO} 4$ indicator solution. Titrate it with standard AgNO3 Titrate to a pinkish yellow end point. Be consistent in end point recognition.

Calculation: [1] $\mathrm{mg} \mathrm{Cl} / \mathrm{L}=(\mathrm{A}-\mathrm{B}) . \mathrm{N} .35450 / \mathrm{ml}$ of sample $(100 \mathrm{ml})$

Where, $A=m l$ titration for sample, $B=m l$ titration for blank, $C=$ normality of $\mathrm{AgNO}_{3}(0.0141 \mathrm{~N})$ [2] $\mathrm{mg} \mathrm{NaCl} / \mathrm{L}=(\mathrm{mg} \mathrm{Cl} / \mathrm{L}) .1 .65$

\subsection{Total Water Hardness}

Take $1 \mathrm{ml}$ of water samples than added few drops of the ammonium bisulphate solution add to black-T as indicator. We observed that water sample color is occurrence pink. Then added EDTA slowly drops by drop and water color is blue.

Calculation: Formula: $1000.1 \mathrm{ml}$ of used in EDTA/ml of water sample.

\subsection{Estimation of Dissolved Oxygen (D.O) and Biological Oxygen Demand (B.O.D)}

$300 \mathrm{ml}$ of B.O.D. bottle was used for water sample Analysis. In this bottle add $1 \mathrm{ml} \mathrm{MnSo} 4$ solution followed by addition of $1 \mathrm{ml}$ alkali iodide acid reagent. Stopper the bottle carefully to exclude and mix by inverting bottle a few times. Add $1 \mathrm{ml}$ concentrated $\mathrm{H}_{2} \mathrm{So}_{4}$. Res topper the bottle and mix it thoroughly too completely dissolve the precipitates. Take $200 \mathrm{ml}$ of this mixture from bottle to flask.

Add $1 \mathrm{ml} 2 \%$ starch solution as indicator. Titrate it with $0.025 \mathrm{Na}_{2} \mathrm{~S}_{2} \mathrm{O}_{3}$ solutions. Record the end point, when the blue color of starch disappears.Calculation: $\mathrm{V}_{1} .0 .1 .1000 / 200$

Where, v1=Burette no.

\subsection{Determination of Acidity of Water}

Hydrogen ions present in a sample as a result of dissociation or hydrolysis of solute react with addition of standard alkali thus acidity depends on end point of the indicator used this colour 
change of phenolphthalein indicator is used to $\mathrm{PH} 8.3$ at $25^{\prime} \mathrm{c}$ response to stoichiometric utilization of carbonic acid to bicarbonate.

Mineral acidity

Volume of NAOH (V1) * N $* 50 * 1000$

Sample taken

Total acidity

Volume of NAOH (V2) $* \quad \mathrm{~N} * 50 \quad * 1000$

Sample taken

\subsection{Determination Alkalinity of Water}

Alkalinity of water can be dermine by titrate in water sample with sulfuric acid or hydrochloric acid based on the reaction and no of moles of hydrochloric acid needed to reach end point conc. Of alkalinity in water is calculated when a water sample that has $\mathrm{pH}$ greater than 4.5 is titrated with acid to a end point of $\mathrm{PH} 4.5$ all ion $\mathrm{oH}-, \mathrm{co} 3$ and HCO3- will be neutralized for the $\mathrm{PH}$ more than 8.3 at phenolphthalein indicator the colour changes to pink colour due to presence of hydroxyl ions on titrating with acid $\mathrm{H}_{2} \mathrm{SO}_{4}$ and HCL. Hydroxyl ions will be neutralized and pink colour will change to colorless. Then add methyl orange colour of the solution with turn to yellow on further titrating with acid the colour will change to red blue to decrease in $\mathrm{pH}$.

Mineral alkalinity

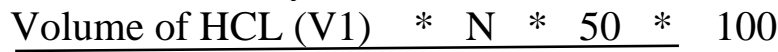

Sample taken

Total alkalinity

Volume of HCL (V2) * N $* 50 * 100$

Sample taken

\section{Results and Conclusion}

Wetland is a great ecosystem and it supports a great Biodiversity. In present work we have investigated interaction between physicochemical parameters with their impact on Microbial biodiversity during pre, middle and post winter time period of 2016-2017 of Barda Bandharan wetland (Temporary wetland) Near Barda Village, Kodinar taluka of Gujarat. Microbial biodiversity is the fourth very important factor of ecosystem in water body because it converts complex organic material into simple organic and inorganic compounds which are utilized by planktons. Water samples collected from wetland of Barda Bandharan around under 2 feet depth of limnetic water. We have included total 15 parameters for analysis like Temperature, $\mathrm{pH}$, Conductivity, T.S, T.D.S., D.O., B.O.D. water Hardness and Chloride. Temperature range was $19.0^{\circ} \mathrm{C}$ to $20.5^{\circ} \mathrm{C}$. $\mathrm{pH}$ range of wetland water was 7.96 to $8.9, \mathrm{pH}$ of water samples were normal Range as per standard but higher $\mathrm{pH}$ of water noted on Nove-11 Month. Higher results of $\mathrm{pH}$ indicated salt concentration may be higher. Conductivity of water was higher in the sample (Dec21), higher conductivity indicates salts concentration may be higher in water samples. Dissolved oxygen (D.O) and Biological oxygen demand (B.O.D) data indicated that dissolve oxygen level 
ranged from 7.9 to 8.9 in water. Higher D.O. value indicated good condition for aquatic life inside the water. T.S. and T.D.S. data of water samples were higher and fluctuated more during time period. T.S. range of sample $1000 \mathrm{mg} / \mathrm{L}$ to $1920 \mathrm{mg} / \mathrm{L}$, Higher, TDS of samples range $300 \mathrm{mg} / \mathrm{L}$ to $490 \mathrm{mg} / \mathrm{L}$ the data of T.S and T.D.S is higher than normal range its indicated water is not directly use for Agriculture and drinking purpose, higher values is also dangerous for normal aquatic life. Water hardness is another parameter which indicated salts quality in water samples like carbonate and many other salts in water sample. Water hardness ranges were $300 \mathrm{mg} / \mathrm{L}$ to $670 \mathrm{mg} / \mathrm{lit}$, salt concentration were increase during sampling time period (Table:01 and Figure:01 to 08)Biological parameters included chlorophyll estimation in which analysis of $\mathrm{Chl} \mathrm{A}, \mathrm{Chl} \mathrm{B}$ and Total Chlorophyll with cartenoids concentration. Chlorophyll play important role in production of organic molecules in water body ecosystem and it's maintaining food web chain in water body ecosystem.

Plankton Analysis was also carried out as Zooplankton and Phytoplankton are very important biotic factor maintaining water body ecosystem. Phytoplankton is primary food producer which is consumed by zooplankton and fish with many other aquatic animals depending on zooplankton concentration in wetland ecosystem. Concentration of chlorophyll also resulted in increased concentration of phytoplanktons. During the present research we have isolated $18 \mathrm{spp}$. of Zooplanktons; Acrocalanus longicornis concentration was higher in which copepod nauplius was predominant in water system. Cylindrotheca spp. was also found during water sample analysis and Cynobacteria were predominantly found in wetland water system.

Table 1: Physico-chemical Analysis of Water sample

\begin{tabular}{|c|c|c|c|c|c|c|c|c|c|c|c|c|}
\hline Date & \multicolumn{3}{|c|}{$11 / 11 / 2016$} & \multicolumn{3}{|c|}{$21 / 12 / 2016$} & \multicolumn{3}{|c|}{$1 / 1 / 2017$} & \multicolumn{3}{|c|}{$10 / 2 / 2017$} \\
\hline $\begin{array}{l}\text { Locati } \\
\text { on }\end{array}$ & 1 & 2 & 3 & 1 & 2 & 3 & 1 & 2 & 3 & 1 & 2 & 3 \\
\hline Time & $8: 42$ & $8: 55$ & 9:07 & $8: 30$ & $8: 47$ & 9:00 & $8: 25$ & $8: 37$ & $8: 52$ & 10:0 & $10: 1$ & $10: 3$ \\
\hline & $\mathrm{AM}$ & AM & $\mathrm{AM}$ & $\mathrm{AM}$ & $\mathrm{AM}$ & AM & $\mathrm{AM}$ & $\mathrm{AM}$ & $\mathrm{AM}$ & $1 \mathrm{AM}$ & $5 \mathrm{AM}$ & $0 A M$ \\
\hline Temp. & 20.0 & 20.2 & 20.5 & 21.0 & 21.5 & 21.8 & 19.5 & 19.0 & 19.2 & 20 & 20.1 & 20.4 \\
\hline Color & $\begin{array}{l}\text { Clea } \\
\mathrm{r}\end{array}$ & $\begin{array}{l}\text { Clea } \\
\mathrm{r}\end{array}$ & $\begin{array}{l}\text { Clea } \\
\mathrm{r}\end{array}$ & $\begin{array}{l}\text { Clea } \\
\mathrm{r}\end{array}$ & $\begin{array}{l}\text { Clea } \\
\mathrm{r}\end{array}$ & $\begin{array}{l}\text { Clea } \\
\mathrm{r}\end{array}$ & $\begin{array}{l}\text { Clea } \\
\mathrm{r}\end{array}$ & $\begin{array}{l}\text { Clea } \\
\mathrm{r}\end{array}$ & $\begin{array}{l}\text { Clea } \\
\mathrm{r}\end{array}$ & Clear & Clear & Clear \\
\hline Order & $\begin{array}{l}\text { Slig } \\
\text { htly } \\
\text { smel } \\
\text { ly }\end{array}$ & $\begin{array}{l}\text { Slig } \\
\text { htly } \\
\text { smel } \\
\text { ly }\end{array}$ & $\begin{array}{l}\text { Slig } \\
\text { htly } \\
\text { smel } \\
\text { ly }\end{array}$ & $\begin{array}{l}\text { Slig } \\
\text { htly } \\
\text { smel } \\
\text { ly }\end{array}$ & $\begin{array}{l}\text { Slig } \\
\text { htly } \\
\text { smel } \\
\text { ly }\end{array}$ & $\begin{array}{l}\text { Slig } \\
\text { htly } \\
\text { smel } \\
\text { ly }\end{array}$ & $\begin{array}{l}\text { Slig } \\
\text { htly } \\
\text { smel } \\
\text { ly }\end{array}$ & $\begin{array}{l}\text { Slig } \\
\text { htly } \\
\text { smel } \\
\text { ly }\end{array}$ & $\begin{array}{l}\text { Slig } \\
\text { htly } \\
\text { smel } \\
\text { ly }\end{array}$ & $\begin{array}{l}\text { Sligh } \\
\text { tly } \\
\text { smell } \\
\text { y }\end{array}$ & $\begin{array}{l}\text { Sligh } \\
\text { tly } \\
\text { smell } \\
y\end{array}$ & $\begin{array}{l}\text { Sligh } \\
\text { tly } \\
\text { smell } \\
\text { y }\end{array}$ \\
\hline $\mathrm{pH}$ & 8.9 & 8.2 & 8.6 & 8.6 & 8.7 & 7.96 & 8.7 & 8.5 & 8.9 & 8.4 & 8.52 & 8.4 \\
\hline $\begin{array}{l}\text { Condu } \\
\text { ctivity }\end{array}$ & $\begin{array}{l}2.35 \\
/ \\
20\end{array}$ & $\begin{array}{l}2.42 \\
/ \\
20\end{array}$ & $\begin{array}{l}2.49 \\
/ \\
20\end{array}$ & $\begin{array}{l}2.76 \\
/ \\
20\end{array}$ & $\begin{array}{l}2.83 \\
/ \\
20\end{array}$ & $\begin{array}{l}2.80 \\
/ \\
20\end{array}$ & $\begin{array}{l}1.90 \\
/ \\
200\end{array}$ & $\begin{array}{l}1.84 \\
/ \\
200\end{array}$ & $\begin{array}{l}1.80 \\
/ \\
200\end{array}$ & $\begin{array}{l}1.83 / \\
200\end{array}$ & $\begin{array}{l}1.84 / \\
200\end{array}$ & $\begin{array}{l}1.90 / \\
200\end{array}$ \\
\hline T.S. & $\begin{array}{l}147 \\
0 \\
\mathrm{mg} / \\
\mathrm{L}\end{array}$ & $\begin{array}{l}156 \\
0 \\
\mathrm{mg} / \\
\mathrm{L}\end{array}$ & $\begin{array}{l}143 \\
0 \\
\mathrm{mg} / \\
\mathrm{L}\end{array}$ & $\begin{array}{l}160 \\
0 \\
\mathrm{mg} / \\
\mathrm{L}\end{array}$ & $\begin{array}{l}140 \\
0 \\
\mathrm{mg} / \\
\mathrm{L}\end{array}$ & $\begin{array}{l}167 \\
0 \\
\mathrm{mg} / \\
\mathrm{L}\end{array}$ & $\begin{array}{l}192 \\
0 \\
\mathrm{mg} / \\
\mathrm{L}\end{array}$ & $\begin{array}{l}183 \\
0 \\
\mathrm{mg} / \\
\mathrm{L}\end{array}$ & $\begin{array}{l}184 \\
5 \\
\mathrm{mg} / \\
\mathrm{L}\end{array}$ & $\begin{array}{l}1000 \\
\mathrm{mg} / \mathrm{L}\end{array}$ & $\begin{array}{l}1010 \\
\mathrm{mg} / \mathrm{L}\end{array}$ & $\begin{array}{l}1500 \\
\mathrm{mg} / \mathrm{L}\end{array}$ \\
\hline T.D.S & $\begin{array}{l}395 \\
\mathrm{mg} / \\
\mathrm{L}\end{array}$ & $\begin{array}{l}370 \\
\mathrm{mg} / \\
\mathrm{L}\end{array}$ & $\begin{array}{l}390 \\
\mathrm{mg} / \\
\mathrm{L}\end{array}$ & $\begin{array}{l}490 \\
\mathrm{mg} / \\
\mathrm{L}\end{array}$ & $\begin{array}{l}300 \\
\mathrm{mg} / \\
\mathrm{L}\end{array}$ & $\begin{array}{l}200 \\
\mathrm{mg} / \\
\mathrm{L}\end{array}$ & $\begin{array}{l}335 \\
\mathrm{mg} / \\
\mathrm{L}\end{array}$ & $\begin{array}{l}342 \\
\mathrm{mg} / \\
\mathrm{L}\end{array}$ & $\begin{array}{l}349 \\
\mathrm{mg} / \\
\mathrm{L}\end{array}$ & $\begin{array}{l}300 \\
\mathrm{mg} / \mathrm{L}\end{array}$ & $\begin{array}{l}350 \\
\mathrm{mg} / \mathrm{L}\end{array}$ & $\begin{array}{l}345 \\
\mathrm{mg} / \mathrm{L}\end{array}$ \\
\hline
\end{tabular}




\begin{tabular}{|c|c|c|c|c|c|c|c|c|c|c|c|c|}
\hline D.O. & $\begin{array}{l}8.9 \\
\mathrm{mg} / \\
\mathrm{L}\end{array}$ & $\begin{array}{l}8.2 \\
\mathrm{mg} / \\
\mathrm{L}\end{array}$ & $\begin{array}{l}8.3 \\
\mathrm{mg} / \\
\mathrm{L}\end{array}$ & $\begin{array}{l}8.1 \\
\mathrm{mg} / \\
\mathrm{L}\end{array}$ & $\begin{array}{l}8.0 \\
\mathrm{mg} / \\
\mathrm{L}\end{array}$ & $\begin{array}{l}7.9 \\
\mathrm{mg} / \\
\mathrm{L}\end{array}$ & $\begin{array}{l}8.7 \\
\mathrm{mg} / \\
\mathrm{L}\end{array}$ & $\begin{array}{l}8.3 \\
\mathrm{mg} / \\
\mathrm{L}\end{array}$ & $\begin{array}{l}8.6 \\
\mathrm{mg} / \\
\mathrm{L}\end{array}$ & $\begin{array}{l}8.7 \\
\mathrm{mg} / \mathrm{L}\end{array}$ & $\begin{array}{l}8.6 \\
\mathrm{mg} / \mathrm{L}\end{array}$ & $\begin{array}{l}8.7 \\
\mathrm{mg} / \mathrm{L}\end{array}$ \\
\hline B.O.D. & $\begin{array}{l}2.5 \\
\mathrm{mg} / \\
\mathrm{L}\end{array}$ & $\begin{array}{l}2.7 \\
\mathrm{mg} / \\
\mathrm{L}\end{array}$ & $\begin{array}{l}2.0 \\
\mathrm{mg} / \\
\mathrm{L}\end{array}$ & $\begin{array}{l}2.0 \\
\mathrm{mg} / \\
\mathrm{L}\end{array}$ & $\begin{array}{l}2.5 \\
\mathrm{mg} / \\
\mathrm{L}\end{array}$ & $\begin{array}{l}2.0 \\
\mathrm{mg} / \\
\mathrm{L}\end{array}$ & $\begin{array}{l}2.7 \\
\mathrm{mg} / \\
\mathrm{L}\end{array}$ & $\begin{array}{l}2.1 \\
\mathrm{mg} / \\
\mathrm{L}\end{array}$ & $\begin{array}{l}2.3 \\
\mathrm{mg} / \\
\mathrm{L}\end{array}$ & $\begin{array}{l}3.2 \\
\mathrm{mg} / \mathrm{L}\end{array}$ & $\begin{array}{l}2.1 \\
\mathrm{mg} / \mathrm{L}\end{array}$ & $\begin{array}{l}3.1 \\
\mathrm{mg} / \mathrm{L}\end{array}$ \\
\hline $\begin{array}{l}\text { Water } \\
\text { hardne } \\
\text { ss }\end{array}$ & $\begin{array}{l}657 \\
\mathrm{mg} / \\
\mathrm{L}\end{array}$ & $\begin{array}{l}670 \\
\mathrm{mg} / \\
\mathrm{L}\end{array}$ & $\begin{array}{l}675 \\
\mathrm{mg} / \\
\mathrm{L} \\
\end{array}$ & $\begin{array}{l}620 \\
\mathrm{mg} / \\
\mathrm{L}\end{array}$ & $\begin{array}{l}664 \\
\mathrm{mg} / \\
\mathrm{L} \\
\end{array}$ & $\begin{array}{l}672 \\
\mathrm{mg} / \\
\mathrm{L} \\
\end{array}$ & $\begin{array}{l}559 \\
\mathrm{mg} / \\
\mathrm{L}\end{array}$ & $\begin{array}{l}500 \\
\mathrm{mg} / \\
\mathrm{L}\end{array}$ & $\begin{array}{l}538 \\
\mathrm{mg} / \\
\mathrm{L} \\
\end{array}$ & $\begin{array}{l}300 \\
\mathrm{mg} / \mathrm{L}\end{array}$ & $\begin{array}{l}310 \\
\mathrm{mg} / \mathrm{L}\end{array}$ & $\begin{array}{l}342 \\
\mathrm{mg} / \mathrm{L}\end{array}$ \\
\hline $\begin{array}{l}\text { Chlori } \\
\text { nity }\end{array}$ & $\begin{array}{l}875 \\
\mathrm{mg} / \\
\mathrm{L}\end{array}$ & $\begin{array}{l}845 \\
\mathrm{mg} / \\
\mathrm{L}\end{array}$ & $\begin{array}{l}835 \\
\mathrm{mg} / \\
\mathrm{L}\end{array}$ & $\begin{array}{l}815 . \\
35 \\
\mathrm{mg} / \\
\mathrm{L}\end{array}$ & $\begin{array}{l}744 . \\
45 \\
\mathrm{mg} / \\
\mathrm{L}\end{array}$ & $\begin{array}{l}890 \\
\mathrm{mg} / \\
\mathrm{L}\end{array}$ & $\begin{array}{l}895 \\
\mathrm{mg} / \\
\mathrm{L}\end{array}$ & $\begin{array}{l}870 \\
\mathrm{mg} / \\
\mathrm{L}\end{array}$ & $\begin{array}{l}855 \\
\mathrm{mg} / \\
\mathrm{L}\end{array}$ & $\begin{array}{l}785 \\
\mathrm{mg} / \mathrm{L}\end{array}$ & $\begin{array}{l}843 . \\
4 \\
\mathrm{mg} / \mathrm{L}\end{array}$ & $\begin{array}{l}852 . \\
4 \\
\mathrm{mg} / \mathrm{L}\end{array}$ \\
\hline $\begin{array}{l}\text { Salinit } \\
\text { y }\end{array}$ & $\begin{array}{l}1.4 \\
\mathrm{~g} / \mathrm{L}\end{array}$ & $\begin{array}{l}1.3 \\
\mathrm{~g} / \mathrm{L}\end{array}$ & $\begin{array}{l}1.3 \\
g / L\end{array}$ & $\begin{array}{l}1.30 \\
\mathrm{mg} / \\
\mathrm{L}\end{array}$ & $\begin{array}{l}1.19 \\
\mathrm{mg} / \\
\mathrm{L}\end{array}$ & $\begin{array}{l}1.40 \\
3 \\
\mathrm{mg} / \\
\mathrm{L}\end{array}$ & $\begin{array}{l}1.43 \\
\mathrm{mg} / \\
\mathrm{L}\end{array}$ & $\begin{array}{l}1.39 \\
\mathrm{mg} / \\
\mathrm{L}\end{array}$ & $\begin{array}{l}1.36 \\
\mathrm{mg} / \\
\mathrm{L}\end{array}$ & $\begin{array}{l}1.27 \\
5 \\
\mathrm{mg} / \mathrm{L}\end{array}$ & $\begin{array}{l}1.24 \\
3 \\
\mathrm{mg} / \mathrm{L}\end{array}$ & $\begin{array}{l}1.32 \\
1 \\
\mathrm{mg} / \mathrm{L}\end{array}$ \\
\hline $\begin{array}{l}\text { Alkalin } \\
\text { ity }\end{array}$ & $\begin{array}{l}3.5 \\
\mathrm{~g} / \mathrm{L}\end{array}$ & $\begin{array}{l}4.3 \\
\mathrm{~g} / \mathrm{L}\end{array}$ & $\begin{array}{l}3.2 \\
\mathrm{~g} / \mathrm{L}\end{array}$ & $\begin{array}{l}3.6 \\
\mathrm{~g} / \mathrm{L}\end{array}$ & $\begin{array}{l}5.1 \mathrm{~g} \\
/ \mathrm{L}\end{array}$ & $\begin{array}{l}3.5 \mathrm{~g} \\
/ \mathrm{L}\end{array}$ & $\begin{array}{l}4.5 \mathrm{~g} \\
/ \mathrm{L}\end{array}$ & $\begin{array}{l}4.0 \mathrm{~g} \\
/ \mathrm{L}\end{array}$ & $\begin{array}{l}4.3 \mathrm{~g} \\
/ \mathrm{L}\end{array}$ & $\begin{array}{l}6.7 \\
\mathrm{~g} / \mathrm{L}\end{array}$ & $\begin{array}{l}6.2 \\
\mathrm{~g} / \mathrm{L}\end{array}$ & $\begin{array}{l}6.3 \\
g / L\end{array}$ \\
\hline Acidity & $\begin{array}{l}3.8 \\
\mathrm{~g} / \mathrm{L}\end{array}$ & $\begin{array}{l}4.2 \\
\mathrm{~g} / \mathrm{L}\end{array}$ & $\begin{array}{l}3.4 \\
\mathrm{~g} / \mathrm{L}\end{array}$ & $\begin{array}{l}3.5 \\
\text { g/L }\end{array}$ & $\begin{array}{l}4.0 \mathrm{~g} \\
/ \mathrm{L}\end{array}$ & $\begin{array}{l}4.3 \mathrm{~g} \\
/ \mathrm{L}\end{array}$ & $\begin{array}{l}4.2 \mathrm{~g} \\
/ \mathrm{L}\end{array}$ & $\begin{array}{l}4.3 \mathrm{~g} \\
/ \mathrm{L}\end{array}$ & $\begin{array}{l}4.1 \mathrm{~g} \\
/ \mathrm{L}\end{array}$ & $\begin{array}{l}2.3 \mathrm{~g} / \\
\mathrm{L}\end{array}$ & $\begin{array}{l}2.4 \\
\mathrm{~g} / \mathrm{L}\end{array}$ & $\begin{array}{l}2.4 \\
\mathrm{~g} / \mathrm{L}\end{array}$ \\
\hline $\begin{array}{l}\mathrm{NaCl} \\
\text { Con. }\end{array}$ & $\begin{array}{l}1.4 \\
\mathrm{~g} / \mathrm{L}\end{array}$ & $\begin{array}{l}1.3 \\
\mathrm{~g} / \mathrm{L}\end{array}$ & $\begin{array}{l}1.3 \\
\mathrm{~g} / \mathrm{L}\end{array}$ & $\begin{array}{l}1.3 \\
\mathrm{~g} / \mathrm{L}\end{array}$ & $\begin{array}{l}1.2 \\
\mathrm{~g} / \mathrm{L}\end{array}$ & $\begin{array}{l}1.4 \\
\mathrm{~g} / \mathrm{L}\end{array}$ & $\begin{array}{l}1.5 \\
\mathrm{~g} / \mathrm{L}\end{array}$ & $\begin{array}{l}1.4 \\
\mathrm{~g} / \mathrm{L}\end{array}$ & $\begin{array}{l}1.41 \\
0 \\
\mathrm{~g} / \mathrm{L}\end{array}$ & $\begin{array}{l}1.29 \\
g / L\end{array}$ & $\begin{array}{l}1.23 \\
\mathrm{~g} / \mathrm{L}\end{array}$ & $\begin{array}{l}1.41 \\
\mathrm{~g} / \mathrm{L}\end{array}$ \\
\hline
\end{tabular}

Table 2(a): Zooplankton variations during sampling time period

\begin{tabular}{|c|c|c|c|c|}
\hline $\begin{array}{c}\text { Barada } \\
\text { Bandharan } \\
\text { (Wetland) }\end{array}$ & $\begin{array}{c}\text { Sampling } \\
\text { Station }\end{array}$ & $\begin{array}{c}\text { Abundance in units } \\
\text { observed/liter }\end{array}$ & $\begin{array}{c}\text { No. of species } \\
\text { observed/total species }\end{array}$ & $\begin{array}{c}\% \text { of } \\
\text { Diversity }\end{array}$ \\
\cline { 2 - 5 } & 1 & 97 & $12 / 20$ & 60.00 \\
\cline { 2 - 5 } & 3 & 113 & $13 / 20$ & 65.00 \\
\hline \multirow{4}{*}{$\begin{array}{c}\text { Bandharan } \\
\text { (Wetland) }\end{array}$} & $\begin{array}{c}\text { Sampling } \\
\text { Station }\end{array}$ & $\begin{array}{c}\text { Abundance in units } \\
\text { observed/liter }\end{array}$ & $\begin{array}{c}\text { No. of species } \\
\text { observed/total species }\end{array}$ & $\begin{array}{c}\% \text { of } \\
\text { Diversity }\end{array}$ \\
\cline { 2 - 5 } & 1 & 112 & $12 / 20$ & 60.00 \\
\cline { 2 - 5 } & 2 & 104 & $14 / 20$ & 70.00 \\
\cline { 2 - 5 } & 3 & 117 & $17 / 20$ & 85.00 \\
\hline $\begin{array}{c}\text { Barada } \\
\text { Bandharan } \\
\text { (Wetland) }\end{array}$ & Stampling & Abundance in units & No. of species & $\%$ of \\
\cline { 2 - 5 } & 1 & observed/liter & observed/total species & Diversity \\
\cline { 2 - 5 } & 2 & 101 & $13 / 20$ & 65.00 \\
\hline
\end{tabular}




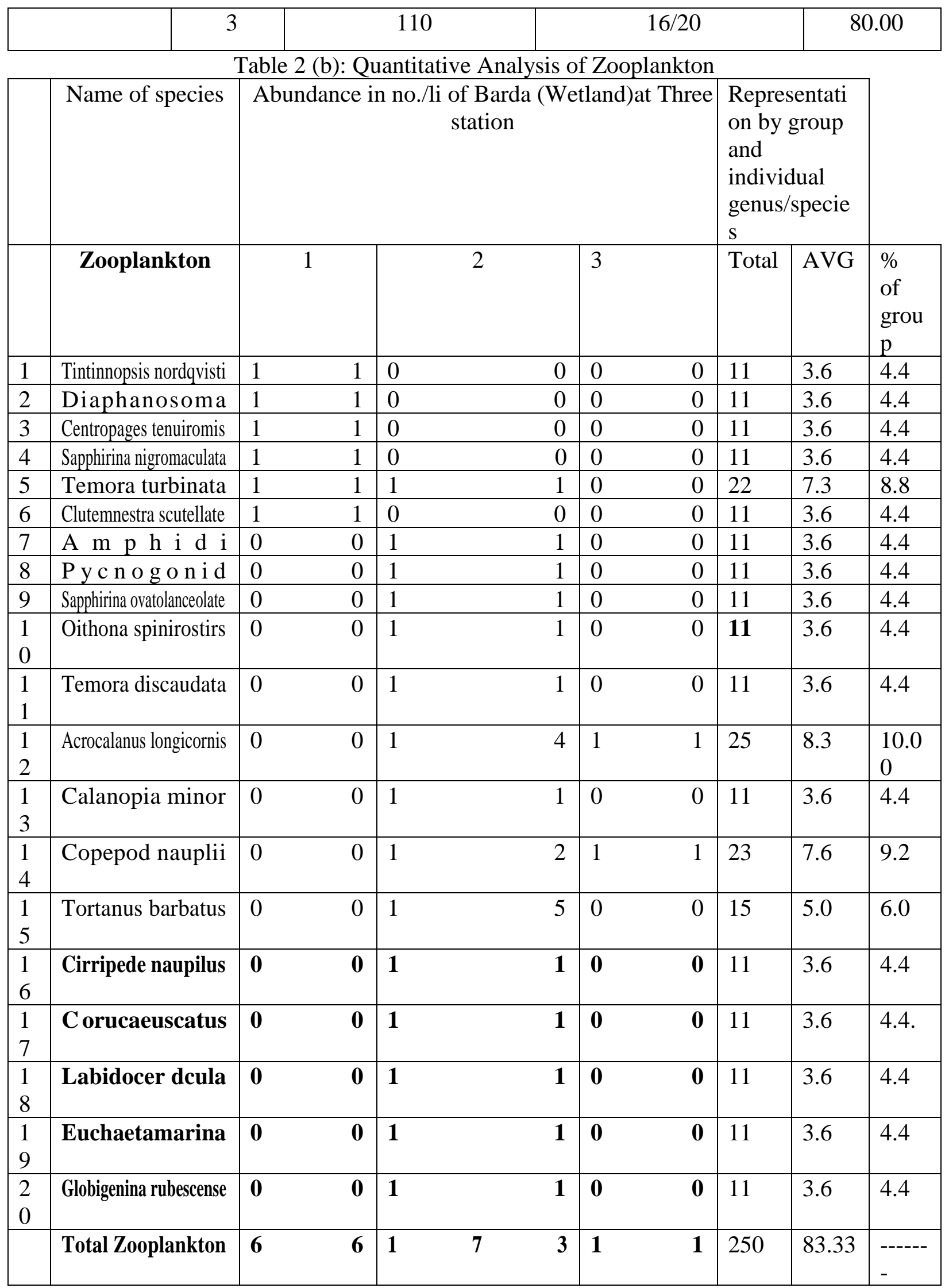




\section{Graphical Data}

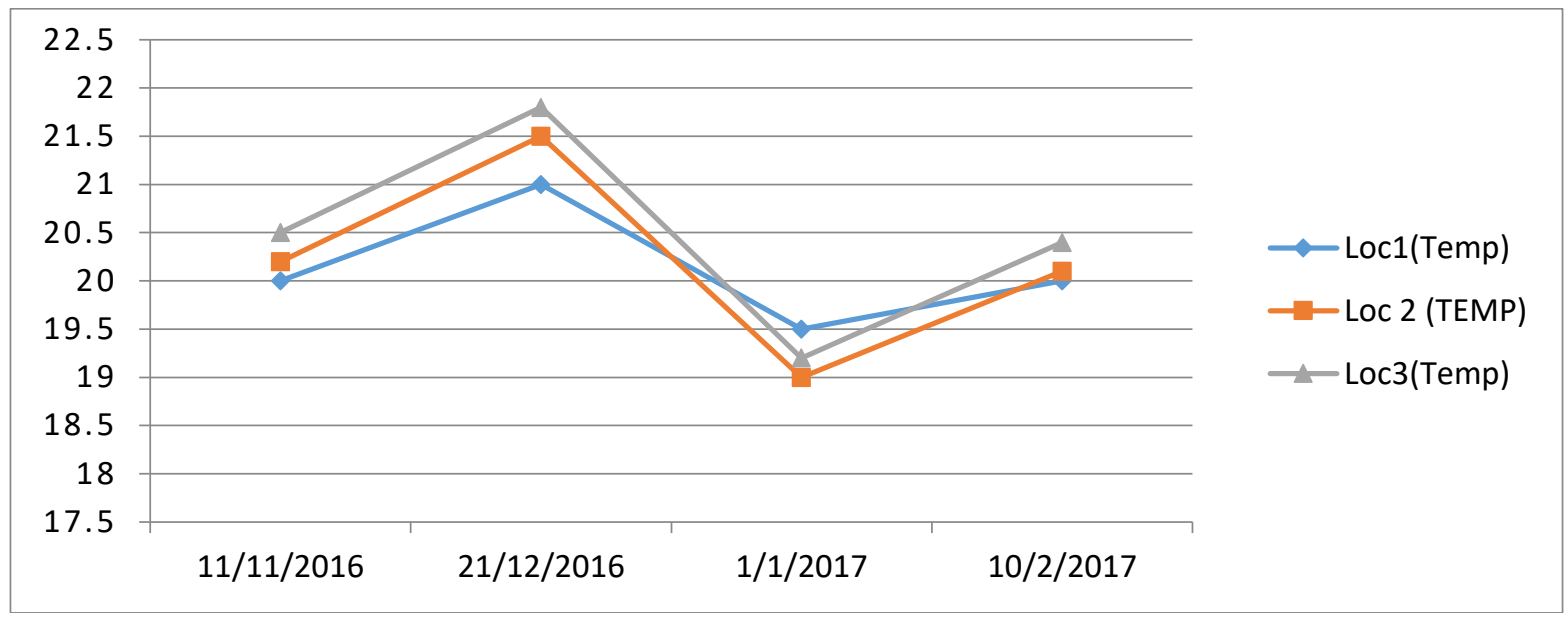

Figure 1: Temperature data Analysis of water sample

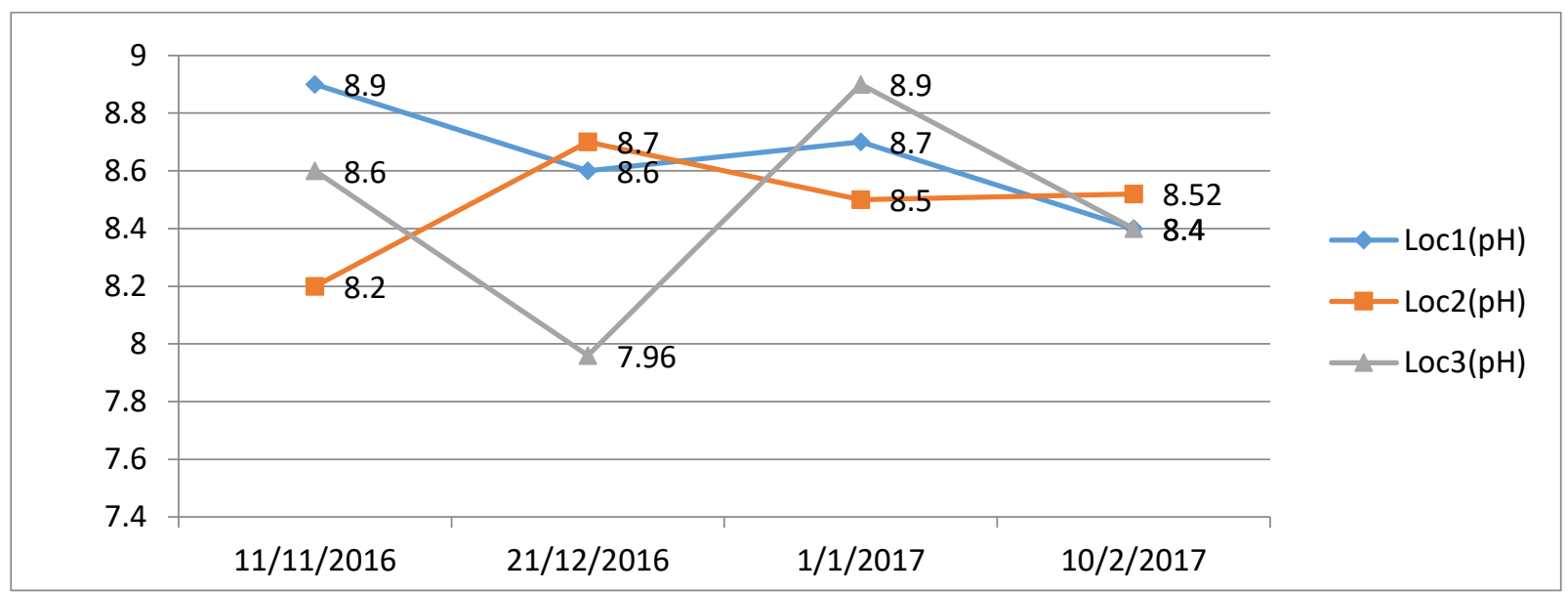

Figure 2: $\mathrm{pH}$ data Analysis of water sample

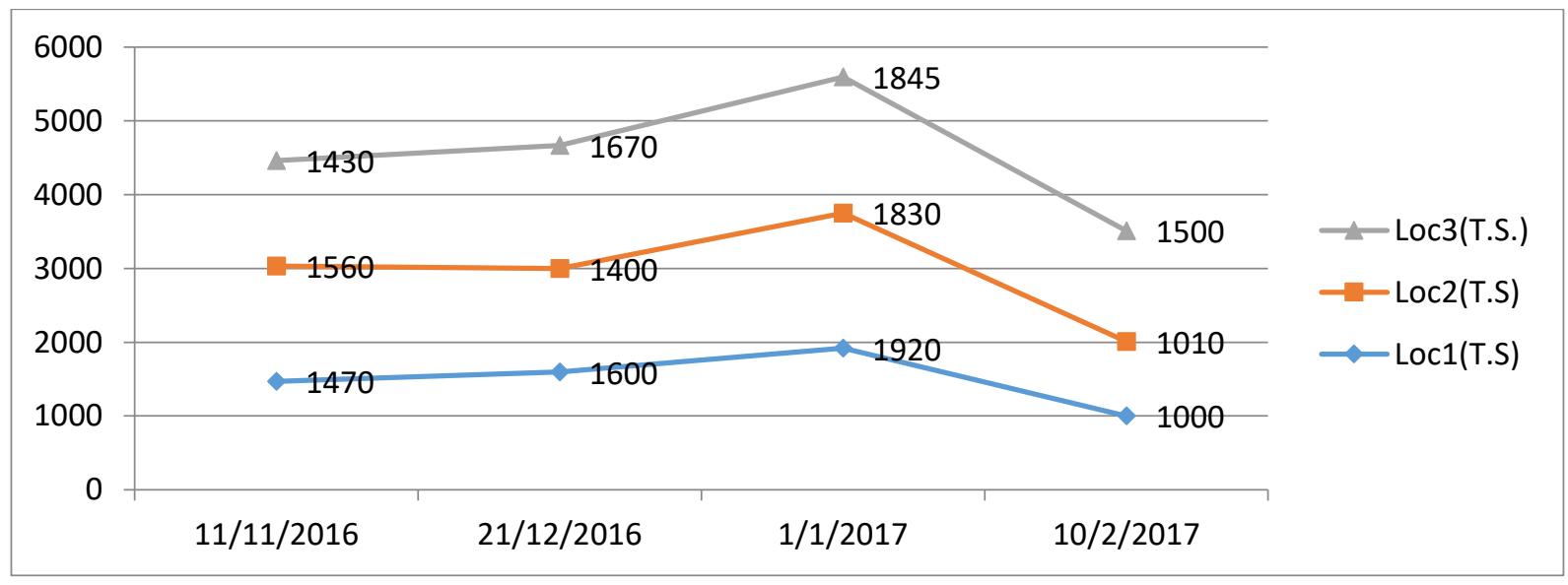

Figure 3: T.S. data Analysis of water sample 


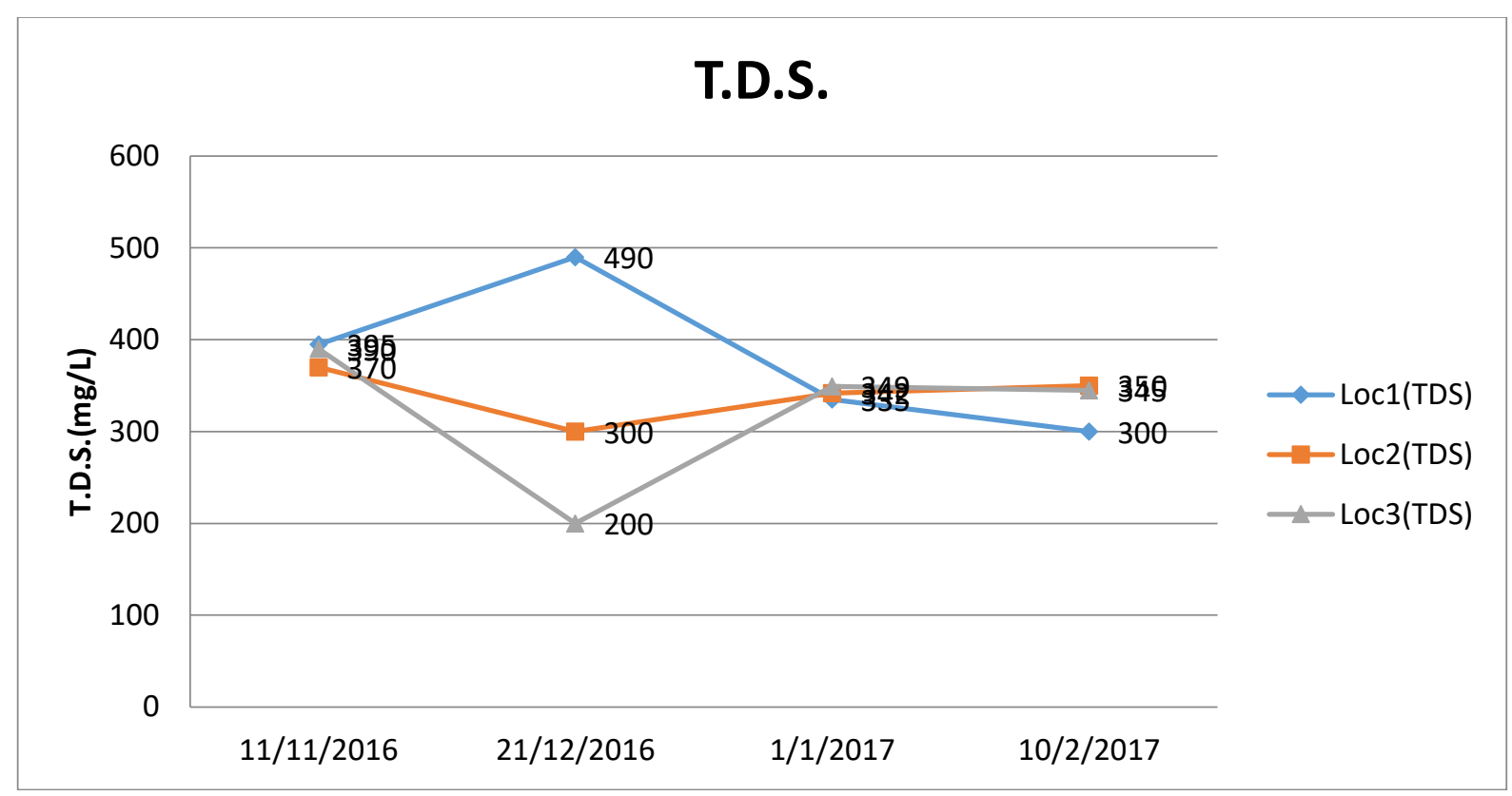

Figure 4: T.D.S. data Analysis of water sample

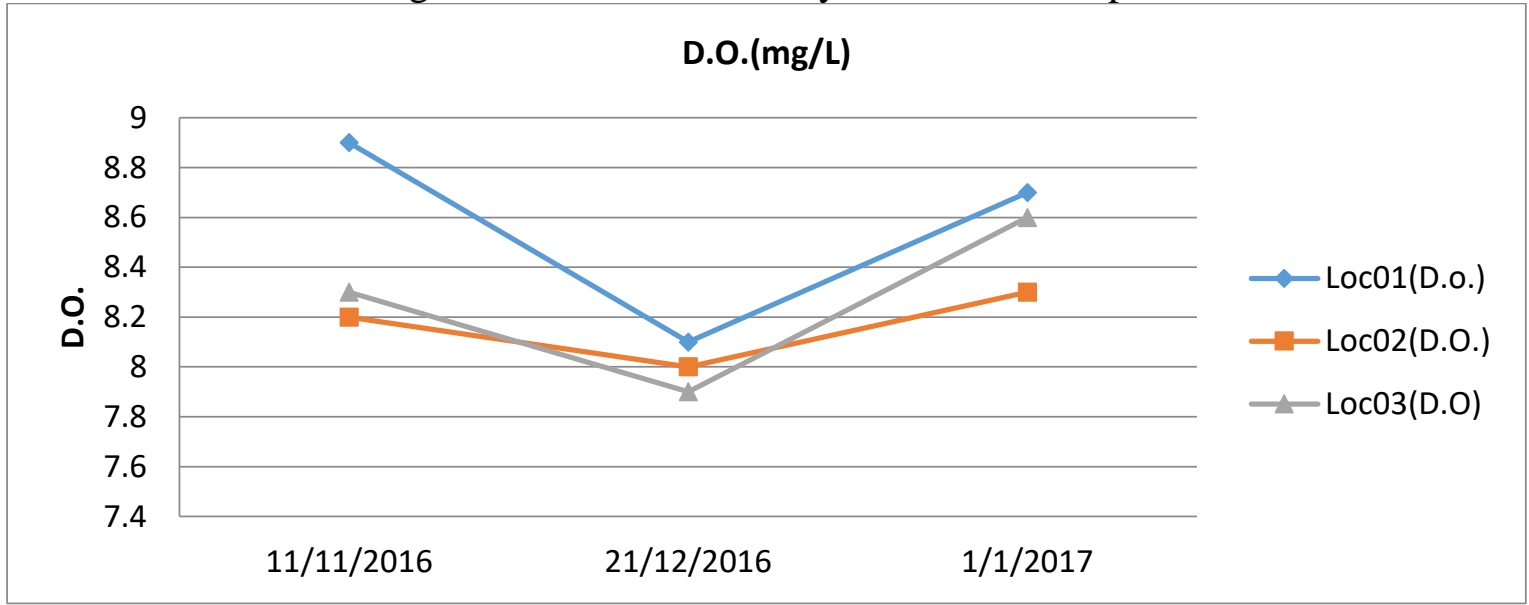

Figure 5: D.O. data Analysis of water sample

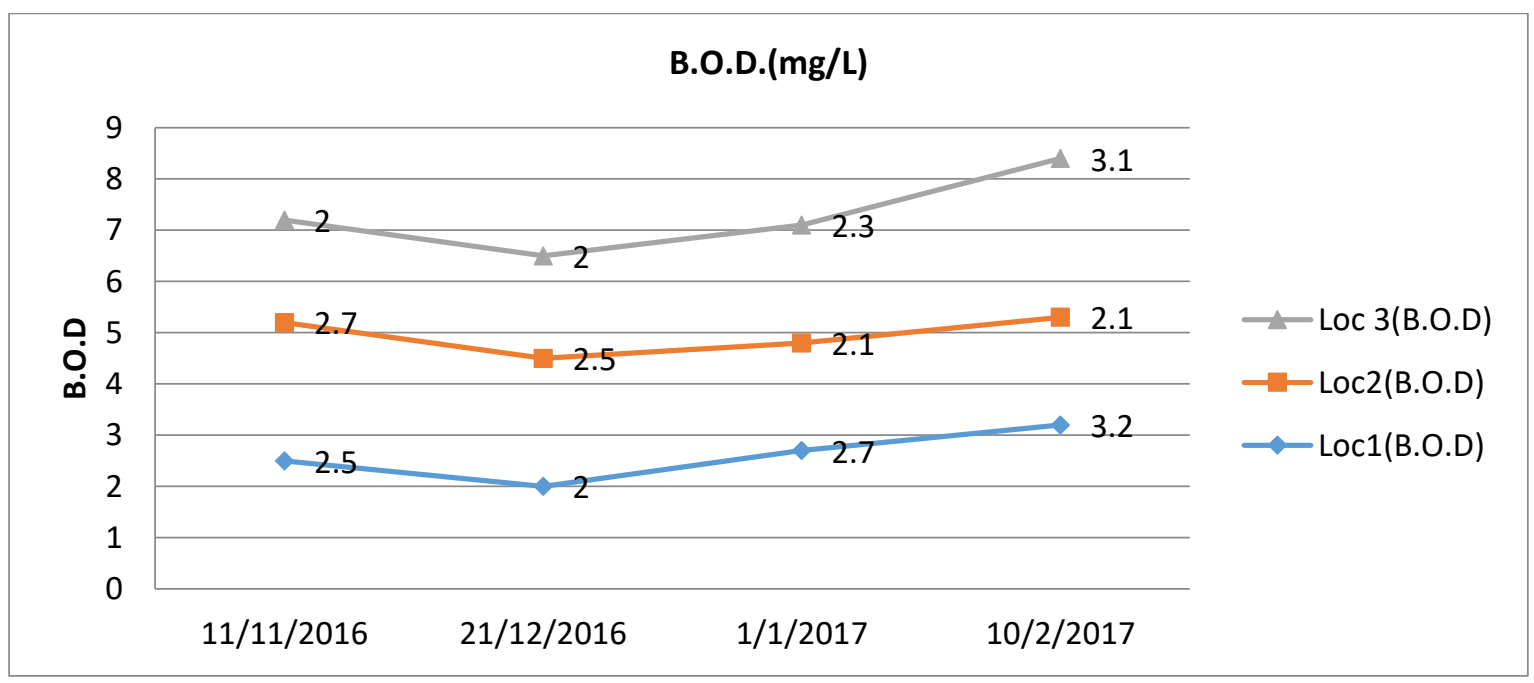


Figure 6: B.O.D. data Analysis of water sample

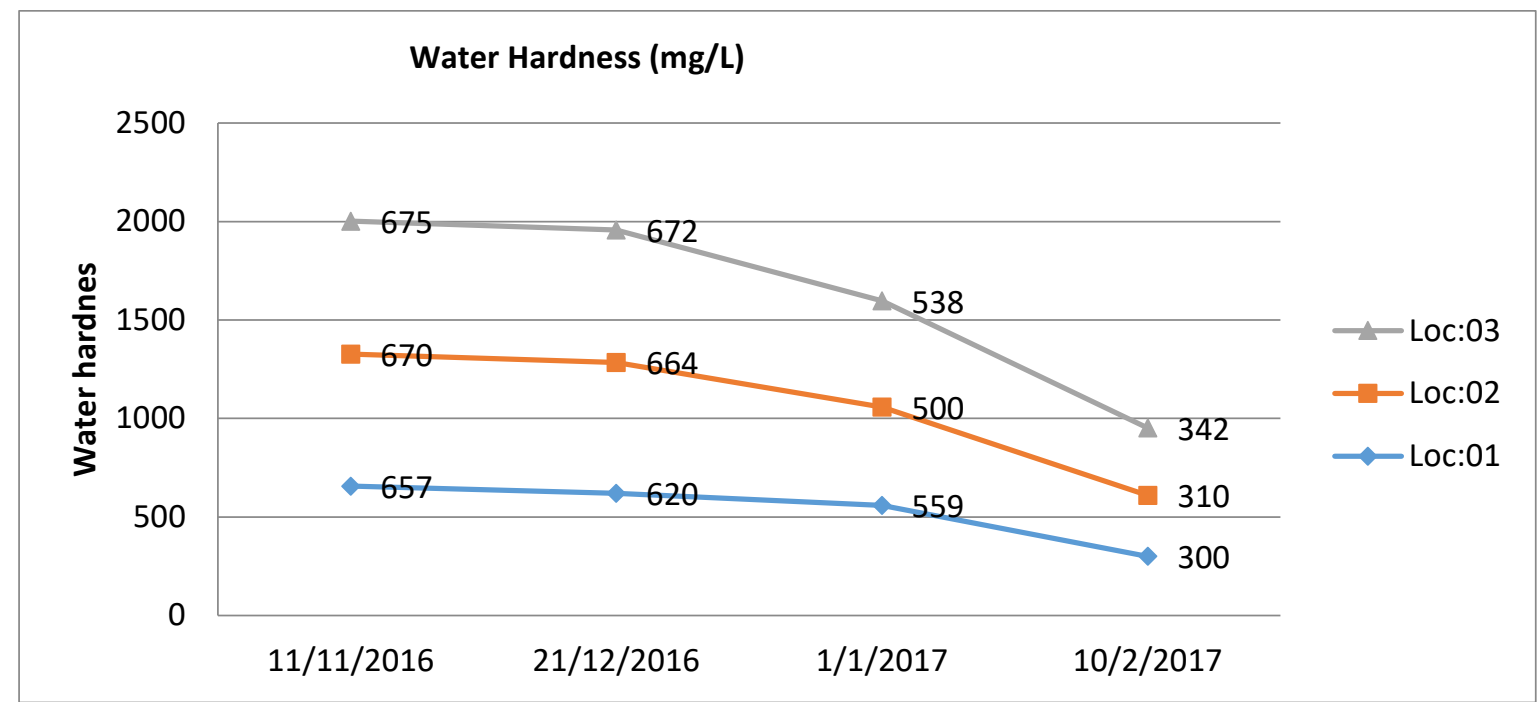

Figure 7: Water Hardness data Analysis of water sample

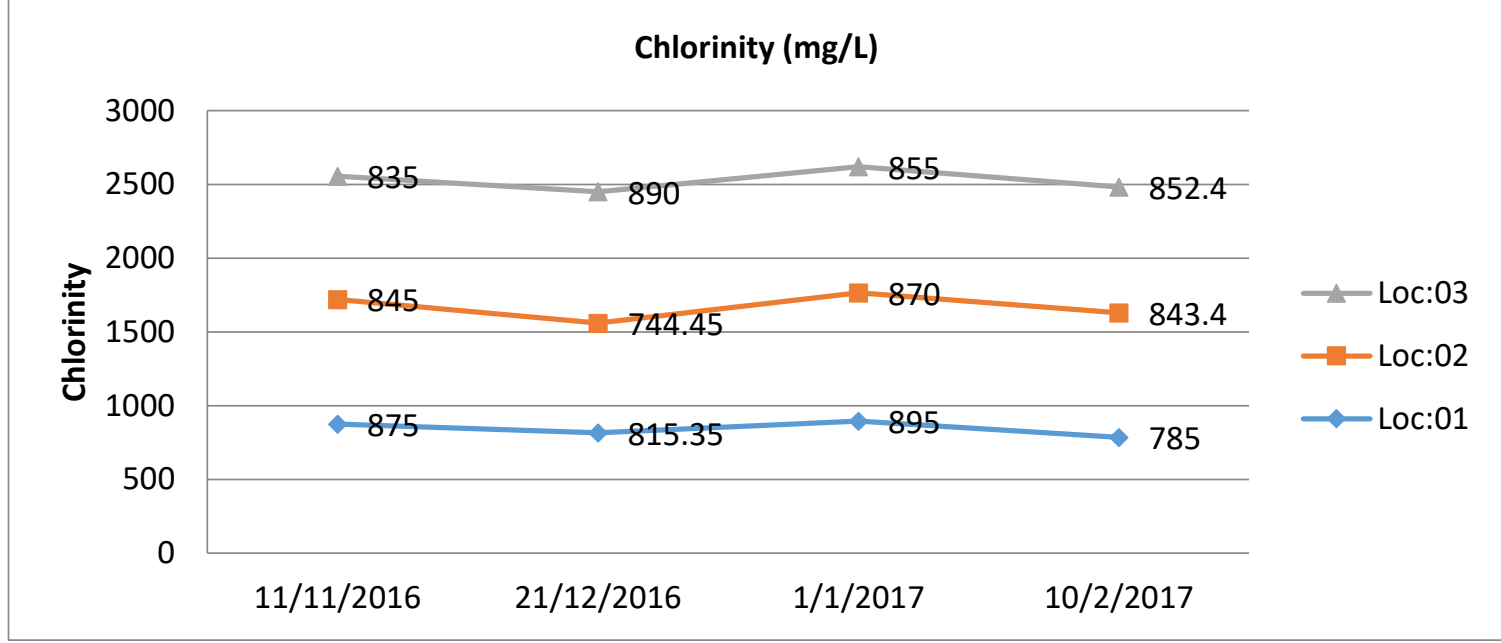

Figure 7: Clorinity data Analysis of water sample

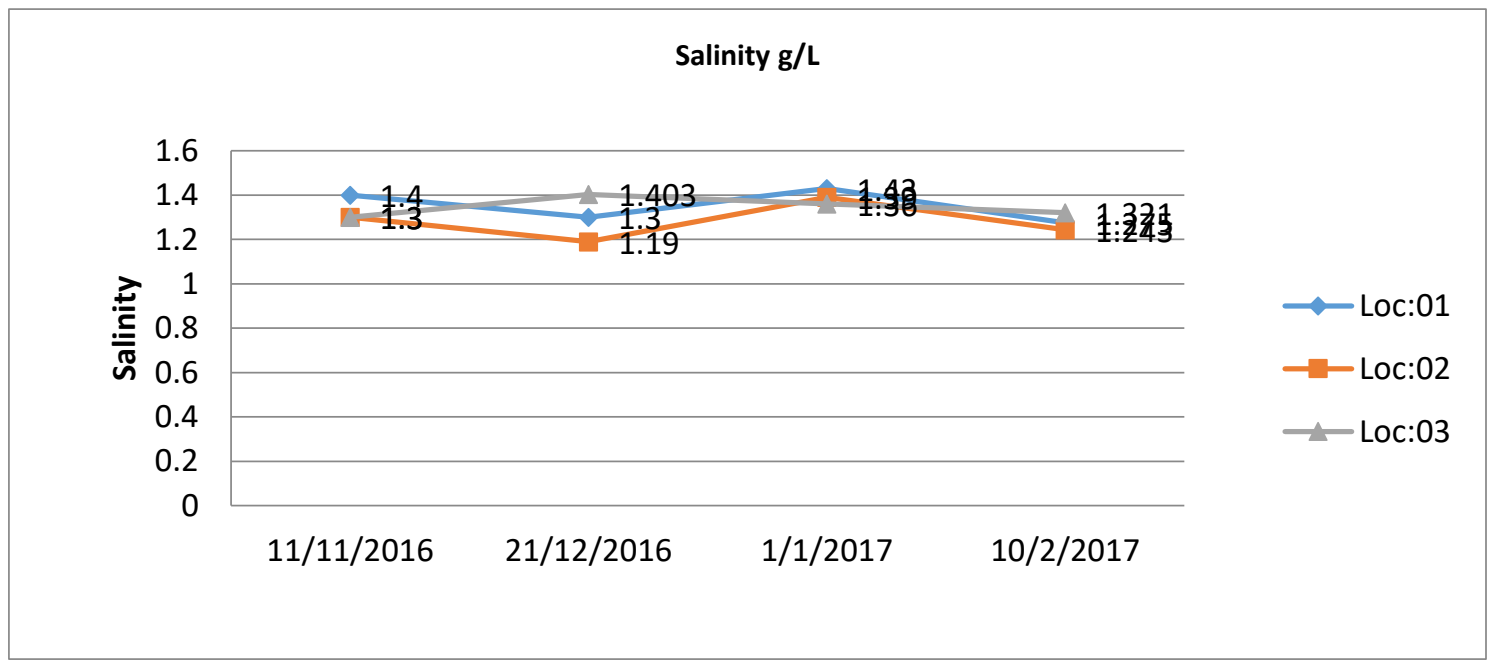


Figure 7: Salinity data Analysis of water sample

\section{Zooplankton Photograph}

Predominant Zooplankton during Water Analysis

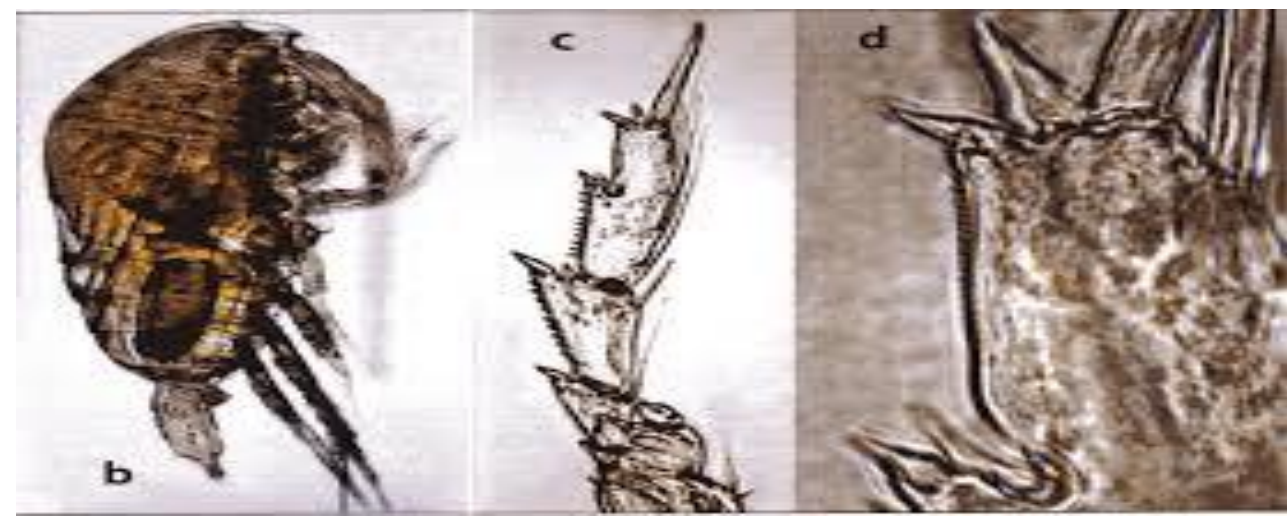

Acrocalanus longicornisspp

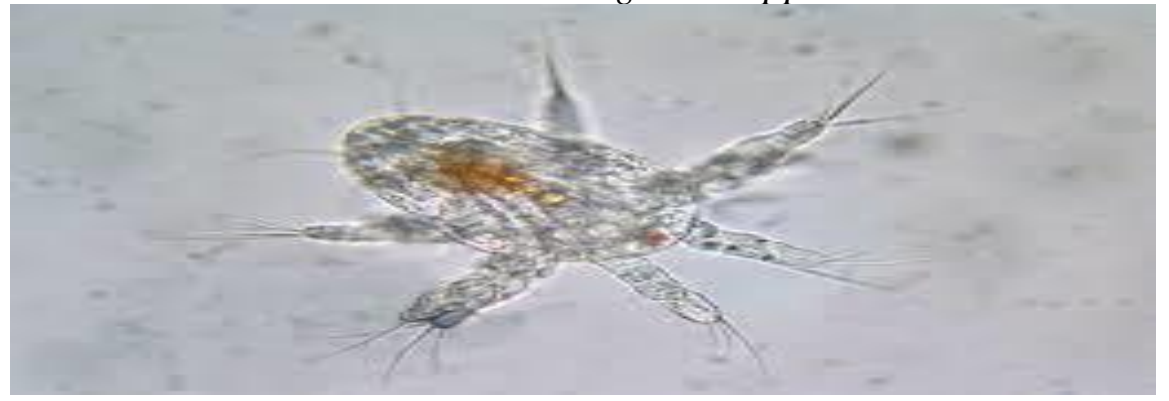

copepod nauplius spp

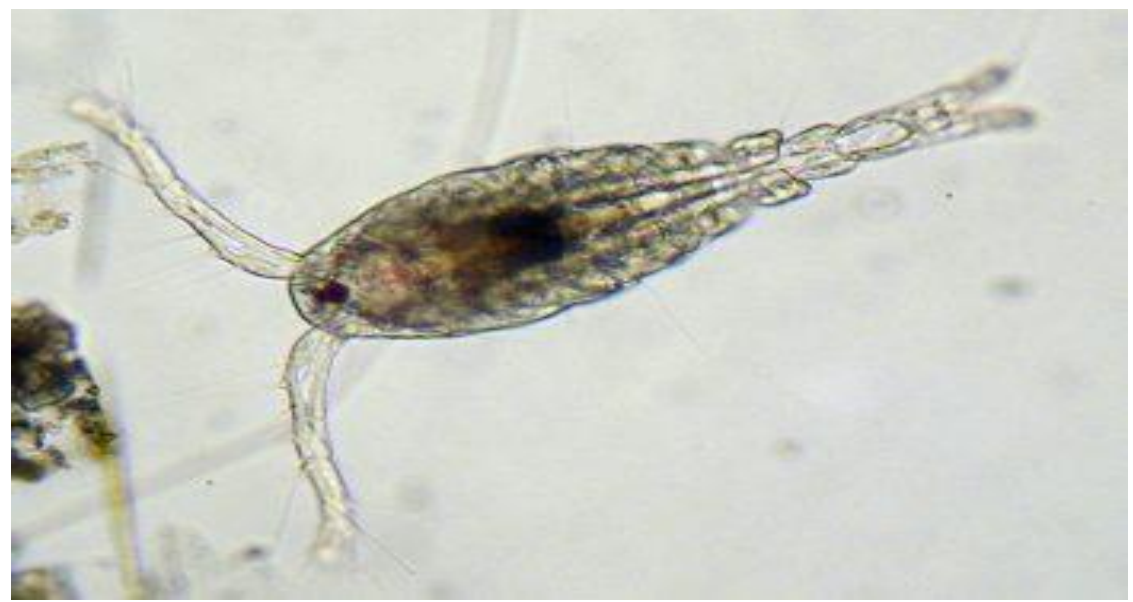

NauplisSpp

\section{References}

[1] American Public Health Association.2012. Standard methods for the examination of water and wastewater. 18th edition. American Public Health Association. Washington, D.C., USA. 
[2] Attayde, J. L., and R. L. Bozelli. 1998. Assessing the in dicator properties of zooplankton assemblages to disturbance gradients by canonical correspondence analysis. Canadian Journal of Fisheries and Aquatic Sciences 55:1789-1797.

[3] Balcer, M. D., N. L. Korda, and S. I. Dodson. 1984. Zooplankton of the Great Lakes. University of Wisconsin Press, Madison, Wisconsin, USA.

[4] Bertram, P., and N. Statler-Salt. 1999. Selection of indicators for Great Lakes basin ecosystem health. Environment Canada and U.S. Environmental Protection Agency. State of the Lakes Ecosystem Conference 1998. [Online, URL: ^ ${ }^{\mathrm{W} w w}$.cciw.ca/solec\&.].

[5] Berzins, B., and J. Bertilsson. 1989. On limnic micro-crustaceans and trophic degree. Hydrobiologia 185:95-100.

[6] Berzins, B., and B. Pejler. 1989. Rotifer occurrence and trophic degree. Hydrobiologia 182:171180.

[7] Botts, P. S. 1999. Lake Erie coastal wetlands: a review and case study of Presque Isle invertebrates. Pages 995-1012in

[8] E. Keas, and C. A. Stricker. 1999. Development of a preliminary invertebrate index of biotic integrity for Lake Huron coastal wetlands. Wetlands 19:869-882.

[9] Campbell, J. M. 1993. The cladoceran species of inshore habitats of Lake Erie at Presque Isle. Journal of the Pennsylvania Academy of Science 67:115-119.

[10] Cardinale, B. J., V. J. Brady, and T. M. Burton. 1998. Changes in the abundance and diversity of coastal wetland fauna from the open water/macrophyte edge towards shore. Wetlands Ecology and Management 6:59-68.

[11] Chow-Fraser, P. 1998. A conceptual model to aid restoration of Cootes Paradise Marsh, a degraded coastal wetland of Lake Ontario, Canada. Wetland Ecology and Management 6:43-57.

[12] Chow-Fraser, P. 1999. Seasonal, inter annual and spatial variability in the concentrations of total suspended solids in a degraded coastal wetland of Lake Ontario. Journal of Great Lakes Research 25:799-813.

[13] Chow-Fraser, P., and D. A. Albert. 1999. Coastal wetland ecosystems: biodiversity investment areas. Environment Canada and U.S. Environmental Protection Agency. state of the Lakes

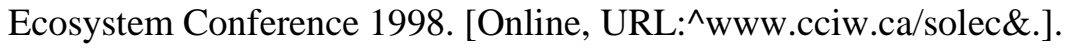

[14] Chow-Fraser, P., V. Lougheed, V. Le Thiec, B. Crosbie, L.Simser, and J. Lord. 1998. Long-term response of the biotic community to fluctuating water levels and changes in water quality in Cootes Paradise Marsh, a degraded coastal wetland of Lake Ontario. Wetland Ecology and Management 6:19-42. 